\title{
Velocity vector imaging to quantify left atrial function
}

\author{
Gabriel Valocik • Ludmila Druzbacká • \\ Ivana Valocikova $\cdot$ Peter Mitro
}

Received: 19 July 2009/Accepted: 9 March 2010/Published online: 26 March 2010

(C) The Author(s) 2010. This article is published with open access at Springerlink.com

\begin{abstract}
The aim of our study was to assess the feasibility of a new image analysis, velocity vector imaging (VVI), in the assessment of left atrial volumes (LAV) and left atrial ejection fraction (LAEF). We retrospectively analysed 100 transthoracic echocardiographic findings in 71 men, and 29 women (mean age $57 \pm 19.8$ years). Two subgroups of patients were defined: (1) with left ventricular (LV) EF $>50 \%$, and (2) LV EF $<50 \%$. For the VVI method of indexed LAV assessment we used the apical four-chamber view. From the displacement of LA endocardial pixels time-volume curves were extracted which provided automatically data regarding indexed maximum LAV (LAVImax), indexed minimum LAV (LAVImin), and LAEF. LAVs and LAEF by 2-dimensional echocardiograhy (2DE) were measured by Simpson's biplane disc summation method. Comparing LAVImax, LAVImin, and LAEF by VVI versus 2DE in the total study population, we found significant correlations: $r=0.94, P<0.0001, r=0.94, P<0.0001, r=$ $0.79, P<0.0001$, respectively. In addition, LAVI$\max \geq 40 \mathrm{ml} / \mathrm{m}^{2}$ was $94 \%$ sensitive and $72 \%$ specific,
\end{abstract}

G. Valocik $(\bowtie) \cdot$ L. Druzbacká · P. Mitro

Third Department of Internal Medicine, Safarik

University Hospital, Kosice, Slovak Republic

e-mail: valocik@central.medic.upjs.sk

I. Valocikova

First Department of Internal Medicine, Safarik University

Hospital, Kosice, Slovak Republic
LAVImin $\geq 27 \mathrm{ml} / \mathrm{m}^{2}$ was $90 \%$ sensitive and $86 \%$ specific, and LAEF $<30 \%$ was $80 \%$ sensitive and 96\% specific for the detection of LV systolic dysfunction. There were highly significant inverse associations of LAVImax and LAVImin to LVEF. LAEF was also significantly related to $\mathrm{LV}$ systolic function. When comparing the time required for VVI and 2DE measurements, VVI led to $62 \%$ reduction in the measurement time. In conclusion, VVI is a feasible method for the assessment of LAVs and LAEF. It provides close agreement with that measured by conventional 2DE Simpson's biplane method with significant time saved.

Keywords Left atrial volume - Left atrial ejection fraction - Velocity vector imaging . Left ventricular systolic function

\section{Introduction}

Left atrial (LA) size is an important predictor of cardiovascular events [1-8]. Different methods exist for the assessment of LA size [9]. The American Society of Echocardiography recommended left atrial volume (LAV) and its indexed value (LAVI) assessed by 2-dimensional echocardiography (2DE), to measure LA size [10]. We undertook this study to determine whether a novel image analysis, known as velocity vector imaging (VVI), can quantify LAV. 


\section{Patients and methods}

We retrospectively analysed 100 transthoracic echocardiographic findings recorded from January to September 2008. Standard transthoracic 2DE and Doppler examinations were performed by one echocardiographer (GV) according to the established clinical laboratory practice using Siemens Sequoia (Siemens Medical Solutions, Inc., Mountain View, California) with an ultrasound transducer operating at $4 \mathrm{~V} 1 \mathrm{c}$ or $3 \mathrm{~V} 2 \mathrm{c}$. During the examination tissue harmonic imaging was used. The images were digitally recorded, stored on magneto-optical discs, and offline analysed. Only echocardiograms of adequate quality were considered. The only exclusion criterion was atrial fibrillation. All the measurements were based on a single measurement of one cardiac cycle. We defined two subgroups of patients: (1) with normal left ventricular $(\mathrm{LV})$ ejection fraction $(\mathrm{EF})>50 \%$, and (2) LV systolic dysfunction, EF $<50 \%$. Left ventricular EF was measured using the modified biplane Simpson's rule. Echocardiographic maximum and minimum left atrial volumes indexed to body surface area (LAVImax and LAVImin) were measured by Simpson's biplane disc summation method [9]. Apical four- and two-chamber views were used at end-systole from the frame immediately preceding mitral valve opening for LAVImax measurement, and at enddiastole, the time at which atrial volume was at its nadir before mitral valve closure, for LAVImin measurement. VVI is a unique technique with complex signal processing but an easy assessment of the timevolume curves $[11,12]$. It requires only a single frame tracing of the endocardial border to extract quantitative time-motion and volume data. The new software (Axius; Siemens Medical Solutions, Inc., Mountain View, California) was used for offline VVI analysis. For the VVI method of LAVI assessment we used the apical four-chamber view. The endocardium of the LA was manually traced starting from the lateral side of the mitral annulus and ending at medial side, excluding the confluence of the pulmonary veins and LA appendage (Fig. 1). We observed tracing the LA at end-systole to provide the best tracking for the cardiac cycle. The velocity vectors superimposed on the $2 \mathrm{DE}$ images were generated. From the displacement of LA endocardial pixels time-volume curves were extracted which provided data regarding LAVmax and LAVmin (Fig. 2). All VVI derived LAV measurements were
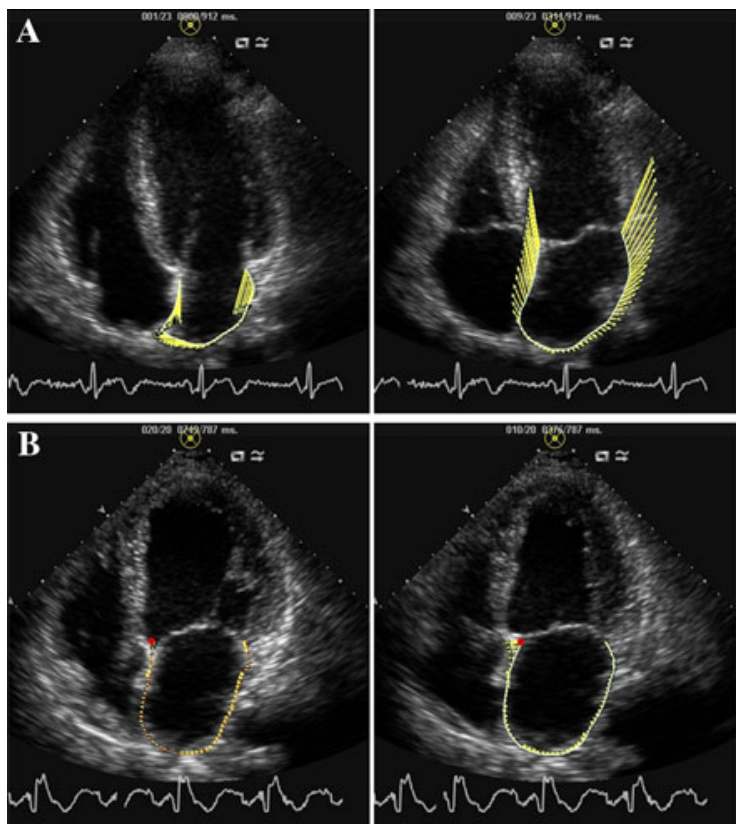

Fig. 1 Assessment of left atrial volumes by vector velocity imaging. After manual tracing of the left atrial endocardium, velocity vectors are generated in cine loop format (left panels - atrial end-systole, right panels-atrial end-diastole). a Patient with normal left ventricular systolic function, b patient with left ventricular systolic dysfunction

indexed to body surface area. Left atrial ejection fraction (LAEF) was calculated automatically by VVI method and in case of $2 \mathrm{DE}$ according to the formula: $\mathrm{LAEF}=\mathrm{LAVmax}-\mathrm{LAVmin} / \mathrm{LAVmax} \times 100 \%$.

In 20 randomly selected patients (10 with normal $\mathrm{EF}$ and 10 with $\mathrm{EF}<50 \%$ ), LAVIs and LAEF by VVI method were reanalysed 1 month later in a blinded fashion for evaluation of intraobserver and interobserver variabilities. In each of these 20 patients the time required for measurements of LAVIs and LAEF by VVI and 2DE was also recorded. The beginning of the measurement was considered the moment of selection of the programme for VVI measurement and volume measurement in case of 2DE. The end of the measurement was considered the moment of acquiring the numerical data.

\section{Statistical analysis}

Data are expressed as mean \pm standard deviation. Significant differences between groups were demonstrated using paired $t$ test. Differences were considered 

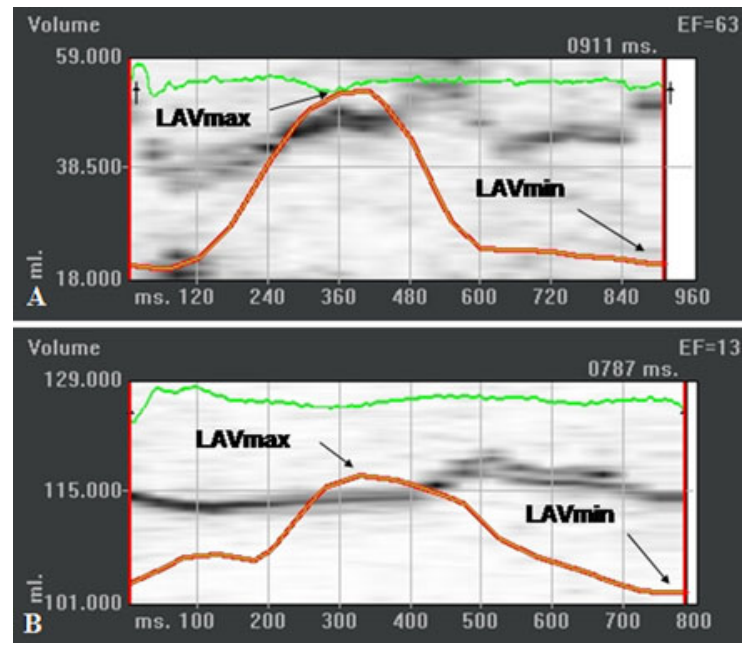

Fig. 2 Time-volume curves extracted from velocity vector imaging. a Patient with normal left ventricular systolic function. b Patient with left ventricular systolic dysfunction. LAVmax - left atrial maximum volume, LAVmin-left atrial minimum volume

statistically significant at a $P$-value of $<0.05$. Linear regression analysis was used to determine the correlation between LAVIs assessed by VVI and 2DE. Analysis of agreement was performed using the method proposed by Bland and Altman [13]. Limits of agreement were defined as mean $\pm 0.95 \times \mathrm{SD}$ of differences. Observer variability was also expressed in correlation coefficient and analysis of agreement. Receiver-operating characteristic curves were constructed to test the ability of LAVIs and LAEF to identify patients with LV systolic dysfunction.

\section{Results}

The study population consisted of 100 patients (71 men, 29 women, mean age $57 \pm 19.8$ years, range
Table 2 Left atrial volumes and ejection fraction using VVI and $2 \mathrm{DE}$

\begin{tabular}{lrrl}
\hline & LV EF $>50 \%$ & LV EF $<50 \%$ & $P$ \\
\hline VVI & & & \\
LAVImax $\left(\mathrm{ml} / \mathrm{m}^{2}\right)$ & $35.13 \pm 14.34$ & $66.67 \pm 19.42$ & $<0.0001$ \\
LAVImin $\left(\mathrm{ml} / \mathrm{m}^{2}\right)$ & $17.45 \pm 11.18$ & $51.34 \pm 18.98$ & $<0.0001$ \\
LAEF $(\%)$ & $52.4 \pm 12.76$ & $24.6 \pm 13.96$ & $<0.0001$ \\
2DE & & & \\
LAVImax $\left(\mathrm{ml} / \mathrm{m}^{2}\right)$ & $30.32 \pm 12.92$ & $57.54 \pm 15.4$ & $<0.0001$ \\
LAVImin $\left(\mathrm{ml} / \mathrm{m}^{2}\right)$ & $19.94 \pm 10.92$ & $48.76 \pm 15.29$ & $<0.0001$ \\
LAEF $(\%)$ & $36.89 \pm 11.17$ & $16.21 \pm 10.41$ & $<0.0001$ \\
\hline
\end{tabular}

Data are expressed as mean $\pm \mathrm{SD}$

$V V I$ velocity vector imaging; LAVImax indexed maximum left atrial volume; LAVImin indexed minimum left atrial volume; $L A E F$ left atrial ejection fraction; $L V E F$ left venctricular ejection fraction; $2 D E 2$ dimensional echocardiography (assesment of the left atrial volume by Simpson's biplane disc summation method)

17-85 years). Baseline clinical characteristics are presented in Table 1. There were highly significant differences in the measured values between the two groups of patients (Table 2).

In the whole group of patients we found an excellent correlation and agreement in LAVIs and moderate correlation in LAEF assessment between VVI and 2DE measurements (Fig. 3).

Patients with normal LVEF (>50\%)

Mean LVEF was $58.82 \pm 6.86 \%$. LAVImax and LAVImin measured by VVI was $35.13 \pm 14.34$ $\mathrm{ml} / \mathrm{m}^{2}$, and $17.45 \pm 11.18 \mathrm{ml} / \mathrm{m}^{2}$, respectively. LAEF was $52.4 \pm 12.76 \%$. LAVImax and LAVImin measured by 2DE Simpson's biplane method were $30.32 \pm 12.92$, and $19.94 \pm 10.92 \mathrm{ml} / \mathrm{m}^{2}$, respectively. LAEF by $2 \mathrm{DE}$ was $36.89 \pm 11.17 \%$ (Table 2 ).

Comparing LAVIs by VVI with LAVIs assessed by $2 \mathrm{DE}$, we found excellent correlations: LAVImax by VVI versus LAVImax by 2DE: $r=0.94$,
Table 1 Baseline clinical characteristics of the total study population and patients with and without left ventricular systolic dysfunction

Continuous data are expressed as mean $\pm \mathrm{SD}$

\begin{tabular}{llll}
\hline Variable & $\begin{array}{l}\text { Total study population } \\
(n=100)\end{array}$ & $\begin{array}{l}\text { LV EF }>50 \% \\
(n=50)\end{array}$ & $\begin{array}{l}\text { LV EF < 50\% } \\
(n=50)\end{array}$ \\
\hline Age (years) & $57.07 \pm 19.87$ & $68.34 \pm 12.54$ & $45.84 \pm 19.52$ \\
Men/women & $63 / 37$ & $23 / 27$ & $40 / 10$ \\
Body surface area $\left(\mathrm{m}^{2}\right)$ & $1.84 \pm 0.19$ & $1.84 \pm 0.21$ & $1.85 \pm 0.18$ \\
Hypertension & $49(49 \%)$ & $15(30 \%)$ & $34(68 \%)$ \\
Diabetes mellitus & $29(29 \%)$ & $6(12 \%)$ & $23(46 \%)$ \\
Coronary artery disease & $48(48 \%)$ & $6(12 \%)$ & $42(84 \%)$ \\
\hline
\end{tabular}



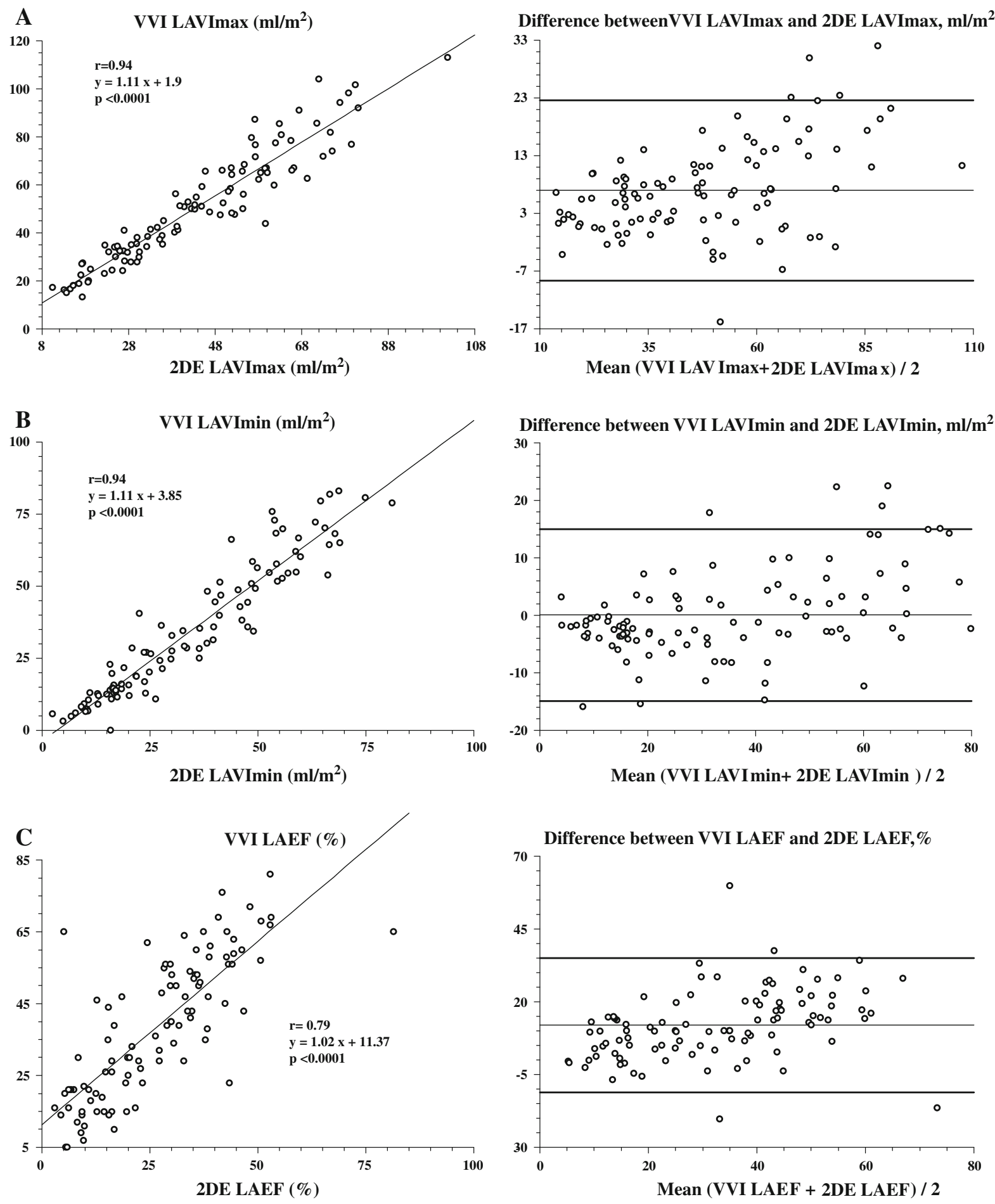

Fig. 3 The results of a linear regression analysis (left panels) and Bland-Altman plots of agreement (right panels) between indexed left atrial maximum volume (LAVImax), indexed left atrial minimum volume (LAVImin), and left atrial ejection

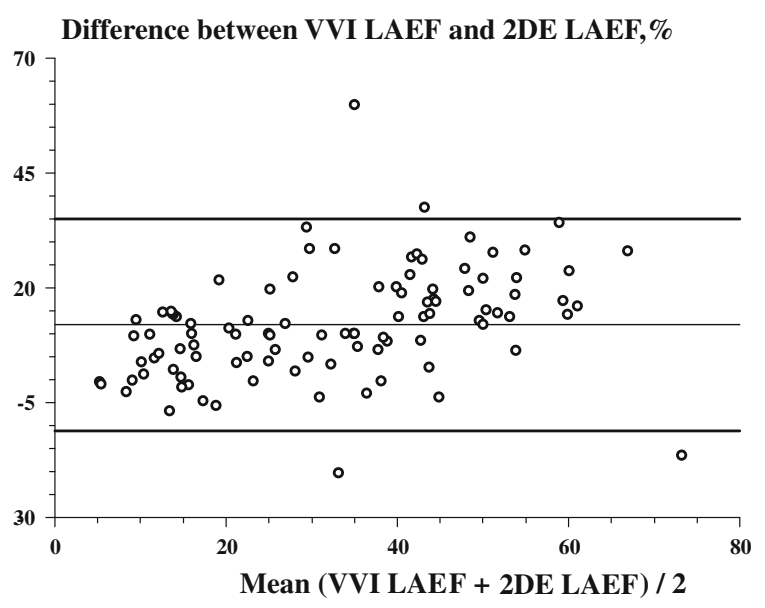

fraction (LAEF) (a, b, and c, respectively) measured by velocity vector imaging (VVI) and 2-dimensional echocardiography (2DE biplane Simpson's method) 
Table 3 Comparison of VVI to 2DE: regression analysis

\begin{tabular}{|c|c|c|c|c|c|c|c|}
\hline & VVI & $r$ & $P$ & $95 \% \mathrm{CI}$ & Regression equation & Mean difference & Limits of agreement \\
\hline \multicolumn{8}{|c|}{ Total study population } \\
\hline LAVImax & & 0.94 & $<0.0001$ & $0.91-0.96$ & $\mathrm{y}=1.11 \mathrm{x}+1.9$ & $6.96 \mathrm{ml} / \mathrm{m}^{2}$ & $-8.62 ; 22.56$ \\
\hline LAVImin & & 0.94 & $<0.0001$ & $0.92-0.96$ & $\mathrm{y}=1.11 \mathrm{x}+3.85$ & $4.58 \mathrm{ml} / \mathrm{m}^{2}$ & $-14.87 ; 14.97$ \\
\hline LAEF & & 0.79 & $<0.0001$ & $0.70-0.85$ & $\mathrm{y}=1.02 \mathrm{x}+11.37$ & $11.94 \%$ & $-11.17 ; 35.06$ \\
\hline \multicolumn{8}{|c|}{$\mathrm{LV} \mathrm{EF}>50 \%$} \\
\hline LAVImax & & 0.94 & $<0.0001$ & $0.90-0.96$ & $y=1.04 x+3.35$ & $4.8 \mathrm{ml} / \mathrm{m}^{2}$ & $-4.55 ; 14.16$ \\
\hline LAVImin & & 0.92 & $<0.0001$ & $0.86-0.95$ & $y=0.94 x+1.35$ & $-2.48 \mathrm{ml} / \mathrm{m}^{2}$ & $-11.05 ; 6.09$ \\
\hline LAEF & & 0.63 & $<0.0001$ & $0.42-0.77$ & $y=0.72 x+25.77$ & $15.5 \%$ & $-4.82 ; 35.83$ \\
\hline \multicolumn{8}{|c|}{$\mathrm{LV} \mathrm{EF}<50 \%$} \\
\hline LAVImax & & 0.86 & $<0.0001$ & $0.77-0.92$ & $\mathrm{y}=1.09 \mathrm{x}+3.75$ & $9.12 \mathrm{ml} / \mathrm{m}^{2}$ & $-10.03 ; 28.29$ \\
\hline LAVImin & & 0.87 & $<0.0001$ & $0.79-0.92$ & $y=1.08 x+1.74$ & $2.57 \mathrm{ml} / \mathrm{m}^{2}$ & $-15.48 ; 20.64$ \\
\hline LAEF & & 0.53 & $<0.0001$ & $0.30-0.70$ & $y=0.71 x+12.97$ & $8.38 \%$ & $-15.43 ; 32.21$ \\
\hline
\end{tabular}

The abbreviations as in Table 2

$P<0.0001$, and LAVImin by VVI versus LAVImin by 2DE: $r=0.92, P<0.0001$. However, comparing LAEF by VVI versus LAEF by $2 \mathrm{DE}$ there was moderate correlation but still highly significant: $r=0.63, P<0.0001$ (Table 3).

Patients with left ventricular systolic dysfunction $(\mathrm{EF}<50 \%)$

Mean LVEF was $31.84 \pm 11.05 \%$. In this group of patients we found significant correlations in LAVI and LAEF assessment, however, the values were less robust as in a group of patients with LVEF $>50 \%$. The data are presented in Table 3.

According to agreement analysis there was systematic overestimation of LAVIs and LAEF by VVI method except of LAVImin in a group of patients with normal LV function (Table 3).

Value of LAVI and LAEF for the detection of LV systolic dysfunction

We examined the sensitivity and specificity of LAVI and LAEF for the detection of systolic dysfunction at different LAVI and LAEF values to develop a receiver-operator characteristic curve. LAVImax $\geq$ $40 \mathrm{ml} / \mathrm{m}^{2}$ was $94 \%$ sensitive and $72 \%$ specific, LAVImin $\geq 27 \mathrm{ml} / \mathrm{m}^{2}$ was $90 \%$ sensitive and $86 \%$ specific, and LAEF $<30 \%$ was $80 \%$ sensitive and $96 \%$ specific for the detection of $\mathrm{LV}$ systolic dysfunction (Fig. 4). C statistics calculated as the area under the receiver-operator characteristic curve were the same for VVI and 2DE (Table 4).

Association of LAVI and LAEF with LV systolic function

There were highly significant inverse associations of LAVImax and LAVImin to LVEF. LAEF was also significantly related to LV systolic function (Fig. 5).

Intraobserver and interobserver variability

The intraobserver correlation coefficient, mean differences and standard errors were: in LAVImax $r=0.97$ $(P<0.0001)$, mean difference $1.94 \pm 12.58(P=$ $0.24)$, standard error 2.81 ; in LAVImin $r=0.96$ $(P<0.0001)$, mean difference $0.51 \pm 11.81(P=$ $0.42)$, standard error 2.64; and in LAEF $r=0.89$ $(P<0.0001)$, mean difference $0.15 \pm 8.71 \quad(P=$ $0.46)$, standard error 1.94 . The interobserver correlation coefficient, mean differences and standard errors were: in LAVImax $r=0.97(P<0.0001)$, mean difference $0.11 \pm 5.05(P=0.46)$, standard error 1.12; in LAVImin $r=0.96(P<0.0001)$, mean difference $-0.45 \pm 5.52(P=0.35)$, standard error 1.23; and in LAEF $r=0.91(P<0.0001)$, mean difference $1.05 \pm 8.16(P=0.28)$ and standard error 1.82 . 

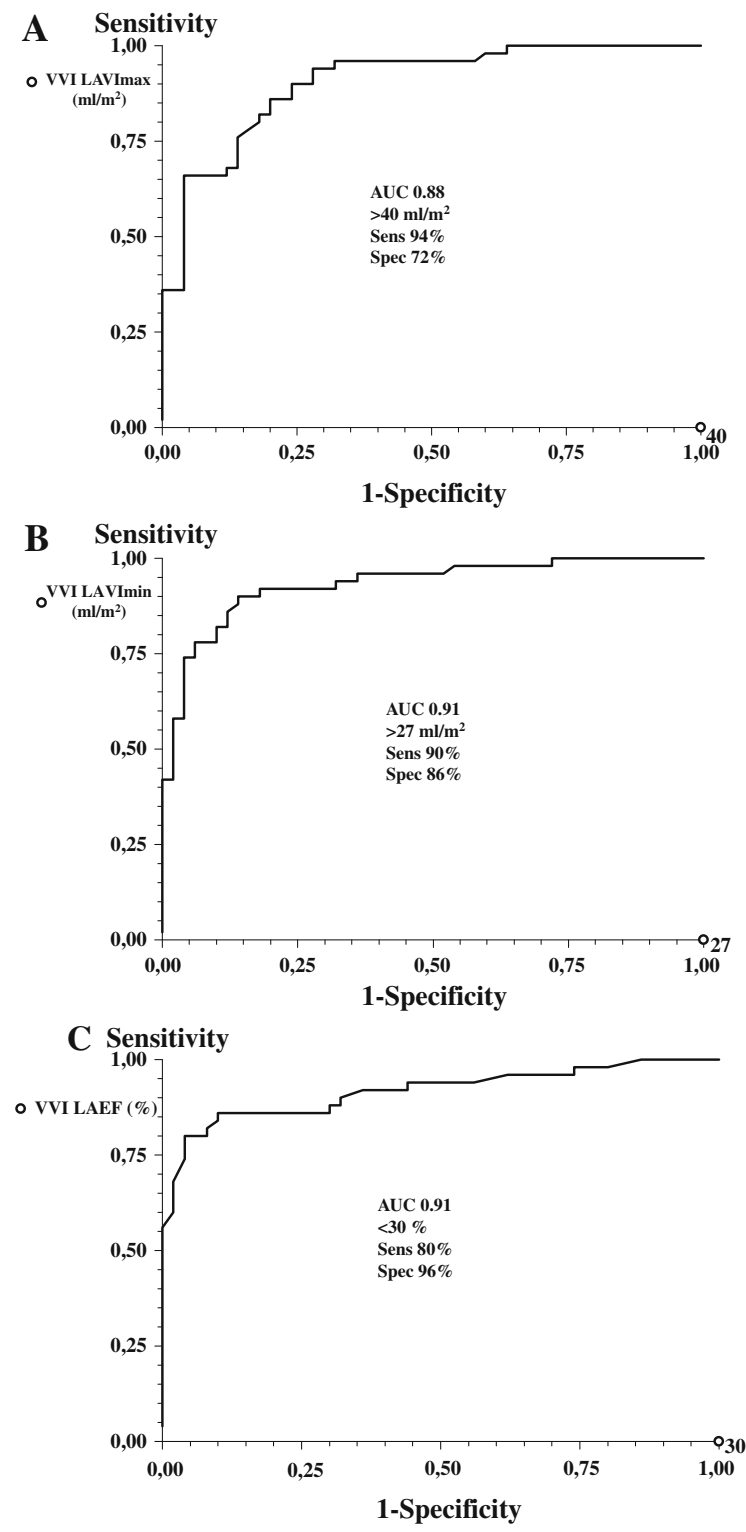

Fig. 4 Receiver-operating characteristic curves for the detection of left ventricular systolic dysfunction. Area under curve (AUC), sensitivity, and specificity for indexed left atrial maximum volume (LAVImax), indexed left atrial minimum volume (LAVImin), and left atrial ejection fraction $(L A E F)(\mathbf{a}, \mathbf{b}$, and $\mathbf{c}$, respectively)
Time required for VVI and 2DE measurements

Mean VVI measurement time was $27.87 \pm 1.09 \mathrm{~s}$ and mean 2DE measurement time was $73.3 \pm 5.29 \mathrm{~s}$. Thus, VVI led to significant $62 \%$ reduction in the measurement time by VVI in comparison with $2 \mathrm{DE}$ $(P<0.0001)$. However, in case of 2DE only LAVImax and LAVImin were automatically provided by the built-in calculation programme. LAEF calculation would require additional time.

\section{Discussion}

Volume of the LA is an important surrogate parameter of the heart function. While LA size assessed by a single linear measurement of the anterior-posterior diameter obtained from M-mode or 2DE has already been shown to be an independent prognostic indicator of cardiovascular events, LAV has been shown to be an even stronger predictor [14-17]. Doppler assessment of the transmitral inflow provides information related to instantaneous pressures in the LV and LA. LAV is a measure of the chronicity and severity of systolic and diastolic dysfunction and is less load dependent. It has been likened to "glycosylated hemoglobin" in the assessment of LV filling pressures.

There are different methods in the assessment of LAV using 2DE (LA planimetry, area-length method, monoplane or biplane Simpson's method) [9]. The American Society of Echocardiography has recommended quantification of LAV by biplane transthoracic echocardiography either using the method of discs (Simpson's rule) or the area-length method [10]. Several studies have proved that LAV assessed by three-dimensional echocardiography, which can be considered as a gold standard in the assessment of LAV, is significantly related to the methods of 2DE. Therefore, 2DE methods of LAV measurements can be used for follow-up evaluation [18-22]. One major

Table 4 Predictive characteristics of LAVIs and LAEF for detection of systolic dysfunction in the study population

\begin{tabular}{llllll}
\hline & $\begin{array}{l}\text { AUC } \\
(\text { VVI })\end{array}$ & $\begin{array}{l}\text { AUC } \\
(2 \mathrm{DE})\end{array}$ & $\begin{array}{l}\text { Optimum cut-off } \\
\text { point }(\mathrm{VVI})\end{array}$ & $\begin{array}{l}\text { Sensitivity } \\
(\text { VVI) }\end{array}$ & $\begin{array}{l}\text { Specificity } \\
\text { (VVI) }\end{array}$ \\
\hline LAVImax & 0.88 & 0.89 & $40 \mathrm{ml} / \mathrm{m}^{2}$ & 0.94 & 0.72 \\
LAVImin & 0.91 & 0.91 & $27 \mathrm{ml} / \mathrm{m}^{2}$ & 0.9 & 0.86 \\
LAEF & 0.91 & 0.91 & $30 \%$ & 0.8 & 0.96 \\
\hline
\end{tabular}

$A U C$ area under receiver-operating characteristic curve (95\% confidence interval); other abbreviations as in Table 2 

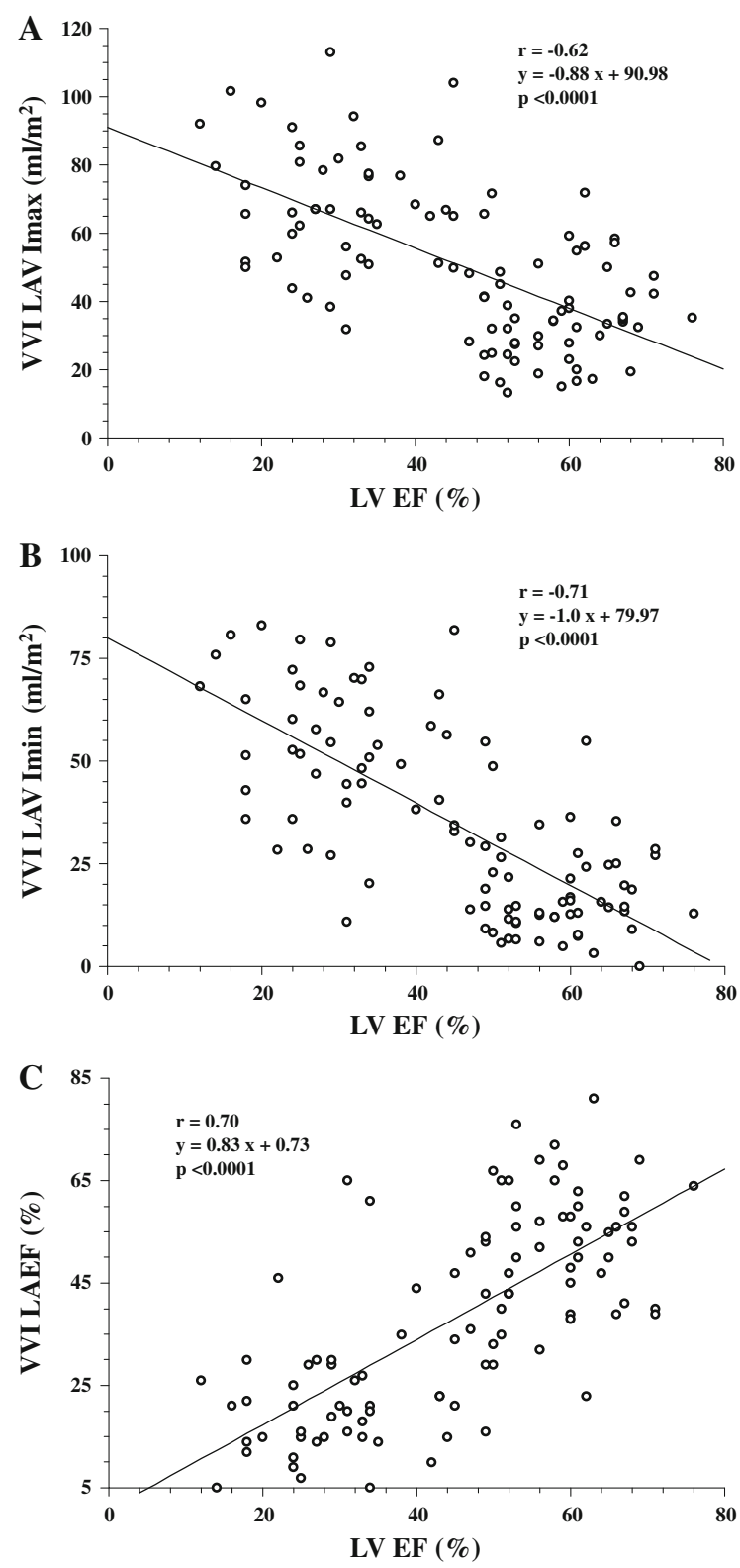

Fig. 5 Association of indexed left atrial maximum volume (LAVImax), indexed left atrial minimum volume (LAVImin), and left atrial ejection fraction $(L A E F)$ with left ventricular ejection fraction $(L V E F)$ (a, b, and $\mathbf{c}$, respectively)

disadvantage is that they are time-consuming procedures not practical in daily clinical practice.

VVI is a novel quantitative echocardiographic technique which requires only a single frame tracing of the endocardial border to extract quantitative timevolume data. The major advantage of VVI is simplicity and short acquisition time. As it was presented in our

study, it provides volume data comparable with the recommended 2DE method of discs. Moreover, VVI led to $62 \%$ reduction in the measurement time in comparison with 2DE.

However, we found an excellent correlation and agreement in LAVIs between VVI and 2DE, for LAEF there was poor to moderate correlation. This may be related to the fact that LAVIs by VVI were overestimated when compared with 2DE. As LAVImax was more overestimated as LAVImin, this resulted in higher LAEF by VVI and so less robust correlation in LAEF by VVI versus 2DE.

In our study we also analysed the relation between LAVI and LV systolic function. LAVIs significantly increased in deteriorating LV systolic function. LAVImax $\geq 40 \mathrm{ml} / \mathrm{m}^{2}$, LAVImin $\geq 27 \mathrm{ml} / \mathrm{m}^{2}$, and LAEF $<30 \%$ were highly sensitive and specific for the detection of LVEF $<50 \%$. The value of LAVI$\max >40 \mathrm{ml} / \mathrm{m}^{2}$ is concordant with the results of the study by Ristow et al. [23] who proved that this volume is predictive to mortality to similar degree as LV systolic dysfunction. Moreover, the American Society of Echocardiography recommends the same value of LAVI $\left(>40 \mathrm{ml} / \mathrm{m}^{2}\right)$ as the cutoff for "severe" LA dilatation [10]. Generelally, only maximum LA size is routinely measured in clinical practice. The prognostic significance of LAVImin and LAEF has not been evaluetad so far. We think that LAVImin and LAEF should have prognostic implication for predicting adverse events in patients with different cardiovascular disorders, analogous to LV end-systolic volume and LVEF. Further studies are needed to prove this assumption.

The major limitation of our study is that we did not use a "gold standard" method (e.g., magnetic resonance imaging, computed tomography) for validation of VVI. Another limitation is that we used VVI in the four-chamber view and compared with $2 \mathrm{DE}$ measurements performed in both four- and two-chamber views. The current VVI method has been designed for only single-plane analysis, although biplane or even three-dimensional analysis would be more accurate for LAV measurements.

The potential benefits of VVI reside in providing not only LAVs but also in evaluation of LA phasic function, so besides LAVImax and LAVImin, assessment of LA reservoir volume, conduit volume, and booster pump volume. In that regard a recent study by Ogawa et al. [24] reported that VVI can be used for 
the rapid and noninvasive automated quantification of LA phasic function. However, the central hypothesis of this study was the assessment of the LA function from time-volume curves, it also demonstrated good agreement between VVI and 2DE in the measurement of LAVs, but in a considerably smaller group of individuals (10 normal subjects and 20 patients). Moreover, speckle-tracking imaging methods have a potential for the assessment of LA strain and strain rate, as it has been recently proposed by several studies [25-27].

\section{Conclusion}

VVI is a feasible method for the assessment of LAVs and LAEF. It provides close agreement with that measured by conventional 2DE Simpson's biplane method with significant time saved. This simplified technique can be readily applied in the clinical setting for reliable and quick quantification of LA enlargement.

Open Access This article is distributed under the terms of the Creative Commons Attribution Noncommercial License which permits any noncommercial use, distribution, and reproduction in any medium, provided the original author(s) and source are credited.

\section{References}

1. Tsang TSM, Barnes ME, Gersh GJ, Bailey KR, Seward JB (2002) Left atrial volume as a morphophysiologic expression of left ventricular diastolic dysfunction and relation to cardiovascular risk burden. Am J Cardiol 90:1284-1289

2. Pritchett AM, Jacobsen SJ, Mahoney DW, Rodeheffer RJ, Bailey KR, Redfield MM (2003) Left atrial volume as an index of left atrial size: a population-based study. J Am Coll Cardiol 41:1036-1043

3. Gottdiener JS, Kitzman DW, Aurigemma GP, Arnold AM, Manolio TA (2006) Left atrial volume, geometry, and function in systolic and diastolic heart failure of persons $>65$ years of age (the Cardiovascular Health Study). Am J Cardiol 97:83-89

4. Pritchett AM, Mahoney DW, Jacobsen SJ, Rodeheffer RJ, Karon BL, Redfield MM (2005) Diastolic dysfunction and left atrial volume. A population-based study. J Am Coll Cardiol 45:87-92

5. Osranek M, Fatema K, Qaddoura F, Al-Saileek A, Barnes ME, Bailey KR et al (2006) Left atrial volume predicts the risk of atrial fibrillation after cardiac surgery. A prospective study. J Am Coll Cardiol 48:779-786

6. De Castro S, Caselli S, Di Angelantonio E, Del Colle S, Mirabelli F, Marcantonio A et al (2008) Relation of left atrial maximal volume measured by real-time $3 \mathrm{D}$ echocardiography to demographic, clinical, and doppler variables. Am J Cardiol 101:1347-1352

7. Tsutsui JM, Dourado PMM, Elhendy A, Falcao SNRS, Goes RM, Chagas ACP et al (2008) Prognostic value of left atrial volume in patients who underwent dobutamine stress echocardiography for known or suspected coronary artery disease. Am Heart J 156:1110-1116

8. Barclay JL, Kruszewski K, Croal BL, Cuthbertson BH, Oh JK, Hillis GS (2006) Relation of left atrial volume to Btype natriuretic peptide levels in patients with stable chronic heart failure. Am J Cardiol 98:98-101

9. Ujino K, Barnes ME, Cha SS, Langins AP, Bailey KR, Seward JB et al (2006) Two-dimensional echocardiographic methods for assessment of left atrial volume. Am J Cardiol 98:1185-1188

10. Lang RM, Bierig M, Devereux RB, Flachskampf FA, Foster E, Pellikka PA et al (2005) Chamber Quantification Writing Group; American Society of Echocardiography's Guidelines and Standards Committee; European Association of Echocardiography. Recommendations for chamber quantification: a report from the American Society of Echocardiography's Guidelines and Standards Committee and the Chamber Quantification Writing Group, developed in conjunction with the European Association of Echocardiography, a branch of the European Society of Cardiology. J Am Soc Echocardiogr 18:1440-1463

11. Kutty S, Deatsman SL, Russell D, Nugent ML, Simpson PM, Frommelt PC (2008) Pulmonary valve replacement improves but does not normalize right ventricular mechanics in repaired congenital heart disease: a comparative assessment using velocity vector imaging. J Am Soc Echocardiogr 21:1216-1221

12. Jurcut R, Pappas CJ, Masci PG, Herbots L, Szulik M, Bogaert $J$ et al (2008) Detection of regional myocardial dysfunction in patients with acute myocardial infarction using velocity vector imaging. J Am Soc Echocardiogr 21:879-886

13. Bland JM, Altman DG (1986) Statistical methods for assessing agreement between two methods of clinical measurement. Lancet 327:307-310

14. Tsang TSM, Abhayaratna WP, Barnes ME, Miyasaka Y, Gersh BJ, Bailey KR et al (2006) Prediction of cardiovascular outcomes with left atrial size is volume superior to area or diameter? J Am Coll Cardiol 47:1018-1023

15. Chinali M, de Simone G, Roman MJ, Bella JN, Liu JE, Lee ET et al (2005) Left atrial systolic force and cardiovascular outcome. Am J Hypertens 18:1570-1576

16. Chinali M, de Simone G, Liu JE, Bella JN, Oberman A, Hopkins PN et al (2005) Left atrial systolic force and cardiac markers of preclinical disease in hypertensive patients: the hypertension genetic epidemiology network (HyperGen) study. Am J Hypertens 18:899-905

17. Kizer JR, Bella JN, Palmieri V, Liu JE, Best LG, Lee ET et al (2006) Left atrial diameter as an independent predictor of first clinical cardiovascular events in middle-aged and elderly adults: the strong heart study (SHS). Am Heart J 151:412-418

18. Anwar AM, Soliman OII, Geleijnse ML, Nemes A, Vletter WB, ten Cate FJ (2008) Assessment of left atrial volume and function by real-time three-dimensional echocardiography. Int J Cardiol 123:155-161 
19. Jenkins C, Bricknell K, Marwick T (2005) Use of threedimensional echocardiography to measure left atrial volume: comparison with other echocardiographic techniques. J Am Soc Echocardiogr 18:991-997

20. Kawai J, Tanabe K, Wang CL, Tani T, Yagi T, Shiotani H et al (2004) Comparison of left atrial size by freehand scanning three-dimensional echocardiography and twodimensional echocardiography. Eur $\mathrm{J}$ Echocardiogr 5: $18-24$

21. Maddukuri PV, Vieira MLC, DeCastro S, Maron MS, Kuvin JT, Patel AR et al (2006) What is the best approach for the assessment of left atrial size? Comparison of various unidimensional and two-dimensional parameters with three-dimensional echocardiographically determined left atrial volume. J Am Soc Echocardiogr 19:1026-1032

22. Singh H, Jain AC, Bhumbla DK, Failinger C (2005) Comparison of left atrial dimensions by transesophageal and transthoracic echocardiography. Echocardiography 22:789-796

23. Ristow B, Ali S, Whooley MA, Schiller NB (2008) Usefulness of left atrial volume index to predict heart failure hospitalization and mortality in ambulatory patients with coronary heart disease and comparison to left ventricular ejection fraction (from the Heart and Soul Study). Am J Cardiol 102:70-76

24. Ogawa K, Hozumi T, Sugioka K, Iwata S, Otsuka R, Takagi Y et al (2009) Automated assessment of left atrial function from time-left atrial volume curves using a novel speckle tracking imaging method. J Am Soc Echocardiogr 22:63-69

25. Cameli M, Caputo M, Mondillo S, Ballo P, Palmerini E, Lisi $M$ et al (2009) Feasibility and reference values of left atrial longitudinal strain imaging by two dimensional speckle tracking. Cardiovasc Ultrasound 7:6

26. Vianna-Pinton R, Moreno CA, Baxter CM, Lee KS, Tsang TS, Appleton CP (2009) Two-dimensional speckle-tracking echocardiography of the left atrium: feasibility and regional contraction and relaxation differences in normal subjects. J Am Soc Echocardiogr 22:299-305

27. Saraiva RM, Demirkol S, Buakhamsri A, Greenberg N, Popovic ZB, Thomas JD et al (2010) Left atrial strain measured by two-dimensional speckle tracking represents a new tool to evaluate left atrial function. J Am Soc Echocardiogr 23:172-180 